Julio César Alonso Cifuentes* Daniela Estrada Nates"

Universidad Icesi Cali, Colombia

Recibido: 8 de mayo de 2018 Concepto de evaluación: 19 de junio de 2018 Aprobado: 22 de junio de 2018

Artículo de investigación (C) 2018 Universidad Católica de Colombia. Facultad de Ciencias Económicas y Administrativas. Todos los derechos reservados

* Doctor en Economía, Profesor y director del Centro de Investigación en Economía y Finanzas (Cienfi), Universidad Icesi, Correo electrónico: jcalonso@icesi.edu.co. https://orcid.org/0000-0003-4890-

7122

** Magíster en Economía, Investigadora del Centro de Investigación en Economía y Finanzas (Cienfi) de la misma universidad. Correo electrónico: destrada@icesi.edu.co. https://orcid.org/0000-0002-4565-

0934.
Finanz. polit. econ., ISSN: 2248-6046, Vol. 10, N. ${ }^{\circ} 2$, julio-diciembre, 2018, pp. 389-402

http://dx.doi.org/10.14718/revfinanzpolitecon.2018.10.2.8

\section{Intentos recientes de redenominación del peso colombiano: una visión histórica*}

\section{RESUMEN}

Este artículo compara los siete proyectos de ley para redenominar la moneda colombiana que han sido radicados en el siglo XXI; con ello se busca contribuir a la escasa literatura sobre el tema y aportar a la discusión del último proyecto de ley radicado en 2018. Para lograrlo, se analizan las propuestas y se narra el paso fallido de los primeros seis proyectos de ley; se encuentra que, si bien los proyectos presentan coincidencias, como la supresión de tres ceros y la creación del nuevo peso, se pueden clasificar en dos grupos específicos. Finalmente, se discuten los elementos necesarios para una evaluación costobeneficio de este tipo de proyectos.

Palabras clave: Colombia, eliminación de ceros, moneda, peso, redenominación monetaria.

JEL: E42, E50

\section{Recent attempts to redenominate the Colombian peso:}

\section{A historical overview}

\section{ABSTRACT}

This paper compares seven bills introduced to the Colombian Congress in the 21st century to redenominate the country's currency. It aims to contribute to the scarce literature on the subject, as well as to the discussion of the bill introduced in 2018. The study presents an analysis of these bills and documents the failed journey of the previous six bills through the Congress. Results show that although there are some coincidences in the proposals, such as dropping three zeros and the creation of the "nuevo peso," the bills can be classified into two main groups. Finally, the paper presents a discussion of the elements necessary for a cost-benefit evaluation of similar bills.

Keywords: Colombia, currency redenomination, currency, dropping zeros, peso.

Una versión detallada de este artículo se encuentra en Alonso, J., Estrada, D. y Gonzáles, A. (2016). Elementos para la discusión de la eliminación de tres ceros en el peso colombiano. En J. Escobar (Ed.), Análisis socioeconómicos: un aporte desde la academia a la actividad legislativa (pp. 29-55). Bogotá: Senado de la República de Colombia. 


\section{Tentativas recentes de redenominação do peso colombiano: uma visão histórica}

\section{RESUMO}

Este artigo compara os sete projetos de lei para redenominar a moeda colombiana propostos no século XXI. Com isso, pretende-se contribuir para a escassa literatura sobre o tema e para a discussão do último projeto de lei radicado em 2018. Para isso, analisam-se as propostas e narra-se o passo falido dos primeiros seis projetos de lei. Constata-se que, embora os projetos apresentem coincidências, como a supressão de três zeros e a criação do novo peso, podem ser classificados em dois grupos específicos. Finalmente, discutem-se os elementos necessários para uma avaliação custo-benefício desse tipo de projetos.

Palavras-chave: Colômbia, eliminação de zeros, moeda, peso, redenominação monetária. 


\section{INTRODUCCIÓN}

El 11 de abril de 2018, el exministro de Hacienda Mauricio Cárdenas radicó ante la Cámara de Representantes el Proyecto de Ley 231/18, que tiene como finalidad eliminar tres ceros del peso colombiano ${ }^{1}$, lo que corresponde al séptimo intento en este siglo por modificar la denominación del peso colombiano. Los proyectos anteriores fueron radicados en 2000, 2002, 2004, 2005, 2010 y 2016, y todos ellos, incluido el nuevo, han tenido como objetivo una reestructuración monetaria: reducir tres ceros en el actual peso, de manera tal que se genere una nueva moneda: "el nuevo peso". Pero la propuesta de cómo hacerlo es relativamente diferente; en algunos casos, el nuevo peso estaría vigente durante un periodo de ajuste que implicaría el retorno a la denominación de peso, mientras que en otras ocasiones la nueva denominación sería permanente. Los proyectos que generan el nuevo peso como la nueva moneda nacional de manera permanente presentan relativamente pocos artículos (cinco o seis), y los que lo crean de manera transitoria son relativamente más (de doce a dieciséis artículos). Este documento presenta una discusión de esos proyectos de ley de reestructuración monetaria en el país, en especial durante este siglo.

En general, la reestructuración monetaria se puede definir, según Odior y Schodeinde (2013), como el proceso que se realiza para asegurar una estructura monetaria óptima, en función de mejorar la eficiencia en las transacciones y en el proceso de emisión de la moneda, lo cual permite tener una mezcla equilibrada de billetes y monedas de varias denominaciones para efectuar las transacciones en el interior de la sociedad. El concepto de reestructuración monetaria así definido es bastante amplio y cubre diferentes medidas como la dolarización, la adopción de una nueva moneda fruto de una unificación monetaria (como el caso de la creación del euro) o la eliminación de ceros. En términos generales, en línea con lo señalado por

1 En la elaboración de este proyecto también participaron el senador Antonio Guerra y el representante a la Cámara Jack Housni, que oficiaban como presidentes de la Comisión Tercera de Cámara y Senado, respectivamente.
Alonso, Estrada y González (2016), un proceso de reestructuración implica por lo menos alguna de las siguientes acciones:

- Adoptar una nueva denominación para la moneda nacional (por ejemplo, pasar de la denominación peso a nuevo peso).

- Cambiar el valor facial de los billetes y las monedas.

- Rediseñar los billetes y las monedas.

La reestructuración de la moneda que implica la eliminación de ceros se conoce como redenominación o currency redenomination. Recientemente, Alonso, Estrada y González (2016) identificaron 91 eventos de redenominación de diferentes monedas alrededor del mundo desde 1923; de hecho, la literatura al respecto no es extensa, y los autores no encontraron algún artículo que presentara una revisión de la literatura sobre el tema, en especial sobre los intentos de reestructuración en Colombia. Dada la nueva propuesta del Ministerio de Hacienda y Crédito Público de eliminar los tres ceros del peso colombiano, este artículo tiene como objetivo actualizar y ampliar el recuento histórico de los intentos recientes de redenominación monetaria en Colombia presentado por Alonso, Estrada y González (2016).

El artículo contiene dos secciones adicionales a esta introducción: en la segunda sección se discuten los intentos recientes de redenominación en Colombia y en la última se presentan los comentarios pertinentes.

\section{LOS INTENTOS RECIENTES DE REESTRUCTURACIÓN EN COLOMBIA}

\section{Breve recuento del siglo XIX y XX}

La primera reestructuración monetaria en Colombia se presentó en 1821 con el nacimiento de la República. El Congreso de Cúcuta, desarrollando el numeral 6 del artículo 55 de la Constitución de 
Cúcuta ${ }^{2}$, creó el peso colombiano, el cual reemplazó al real con una tasa de convertibilidad de ocho reales por un peso (Alonso, Estrada y González, 2016; Torres, 1980). En 1871, el Congreso aprobó adoptar el patrón oro y anclar el peso al franco francés, empleando una tasa de convertibilidad de cinco francos por un peso; es decir, se aprobó una redenominación de la moneda que clasificaría hoy en día en la categoría de dolarización.

Posteriormente, la Junta de Amortización del Banco Nacional de la República (creada en 1880) generó otro evento de redenominación: el papel moneda fue convertido en el peso oro, empleando una tasa de convertibilidad de 100 pesos papel moneda por cada peso oro (Ibáñez, 1991; Avella, 1987; Torres, 1980), que finaliza en 1931 mediante el Decreto-Ley 1683.

Los dos últimos intentos de redenominación del siglo XX se dieron en 1993: el primero con el propósito de adoptar una nueva denominación para la moneda colombiana: el Santander (Alonso, Estrada y González, 2016); el segundo consistía en modificar la denominación de la moneada a nuevo peso y eliminar tres ceros, la primera redenominación. Ambas iniciativas fueron archivadas.

\section{Los intentos de redenominación en el siglo XXI}

Durante lo corrido del siglo XXI se han radicado en el Congreso siete proyectos de redenominación del peso colombiano. En la gráfica 1 se presenta la línea de tiempo de estos proyectos. A continuación, se destacan los aspectos más importantes de cada proyecto y su trámite por el Congreso.

El 22 de agosto del 2000 los senadores José Jaime Nicholls (Movimiento Defensa Ciudadana), Piedad Esneda Córdoba (Partido Liberal Colombiano) y Francisco Javier Murgueitio (Partido Conservador Colombiano) radicaron el Proyecto de Ley 74 de 2000, Senado, que buscaba "modificar la denominación de la moneda

2 El artículo le daba la facultad al Congreso de "determinar y uniformar el valor, peso, tipo y nombre de la moneda". legal colombiana, en desarrollo del numeral 13 del artículo 150 de la Constitución Política". La nueva moneda se llamaría el nuevo peso y circularía de manera paralela a los billetes y las monedas denominados en pesos por periodo de transición; tendría además una tasa de convertibilidad de 1000 pesos por cada nuevo peso.

Una vez se realizara la transición completa al nuevo peso, se haría la transición de este al peso con una equivalencia de un nuevo peso a un peso. El proyecto constaba de doce artículos. El primer artículo creaba el nuevo peso de manera transitoria y otorgaba poder liberatorio ilimitado al nuevo peso colombiano mientras coexistiera con el peso; es decir, cualquier deuda se podría pagar con cualquiera de las nuevas denominaciones de los billetes expresados en nuevos pesos, igual que los billetes actuales, durante el periodo de transición; además, establecía el símbolo "N\$" para la nueva unidad y el "Cn" para los centavos.

El segundo artículo le permitía al Banco de la República seguir emitiendo billetes y monedas denominados en pesos durante el periodo de transición y lo obligaba a facilitar la convertibilidad. El tercer artículo establecía que todos los precios deberían denominarse en nuevos pesos. El cuarto implicaba que todas las obligaciones que estuviesen previamente expresadas en pesos se convertirían a nuevos pesos. El quinto establecía que todos los contratos $u$ obligaciones que estaban en dólares $u$ otra moneda extranjera, y se tuviesen que enunciar en moneda local, se expresaran, desde la sanción de la Ley, en nuevos pesos. El sexto artículo aseguraba que las obligaciones pasadas serían expresadas en nuevos pesos y establecía cómo se realizaría el redondeo. El séptimo estipulaba que una vez todos los billetes y monedas denominados en pesos saliesen de circulación, y cuando la Junta del Banco de la República considerase que la economía colombiana estaba en condiciones de hacer una transición a una nueva denominación, el nuevo peso tomaría nuevamente el nombre de peso.

El artículo octavo determinaba que las reglas para pasar de nuevos pesos a pesos serían las mismas empleadas para pasar inicialmente de pesos 
Linea de tiempo de proyectos de redenominación del siglo XXI

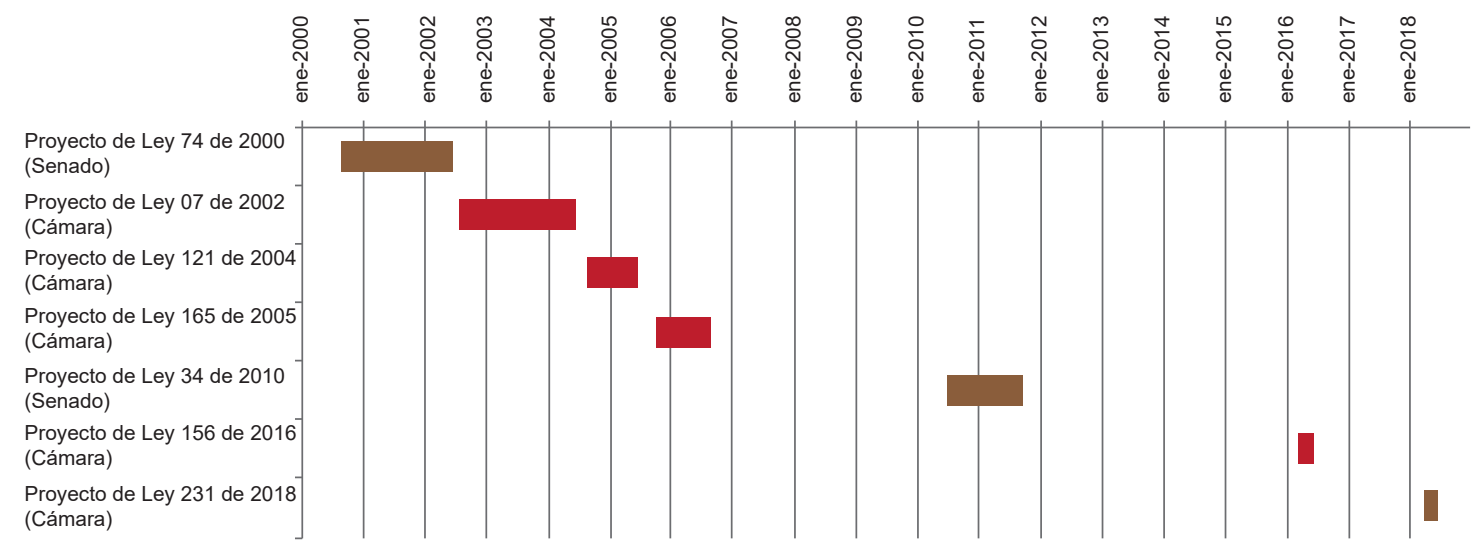

Nota: la longitud de la barra indica el tiempo que transcurrió entre la fecha de radicación del proyecto y la fecha en la que se archivó, excepto para el Proyecto de Ley 231 de 2018, que sigue siendo discutido en el Congreso.

Fuente: elaboración de los autores.

a nuevos pesos. El artículo noveno garantizaba que todas las cifras expresadas en leyes o actos administrativos que estuviesen expresadas en moneda nacional serían cambiadas de acuerdo con la redenominación de la moneda. El artículo décimo garantizaba que las resoluciones judiciales que se expidiesen después de un mes de promulgada la Ley se empezarían a denominar en nuevos pesos. El artículo once se refería a la fecha en la que entraría en vigor la ley y el último artículo derogaba todas las disposiciones contrarias a esta ley.

Los argumentos para sustentar el Proyecto de Ley 74 de 2000 eran que esta reestructuración monetaria mejoraría la eficiencia del sistema financiero y contable; se contempló allí que la eliminación de los ceros permitiría "lograr el uso más eficiente de los sistemas de cómputo, donde la digitación de grandes cifras toma tiempo, aumenta la posibilidad de errores y ocupa espacio valioso en los equipos y archivo". Adicionalmente, se argumentaba que la nueva moneda permitiría consolidar la confianza interna, dada la baja inflación que el país había alcanzado. El 24 de noviembre del 2000, Miguel Urrutia, gerente del Banco de la República, y Juan Manuel Santos, ministro de Hacienda de la época, expresaron su apoyo al proyecto de Ley.
El proyecto pasó tres debates: uno en la Comisión Tercera del Senado, otro en la plenaria del Senado y el último en la Comisión Tercera de la Cámara. El 18 de junio de 2002, el proyecto fue archivado tras la votación mayoritariamente negativa en la plenaria de la Cámara en su cuarto debate. Durante su trámite por el Senado y la Cámara se le incluyó una fórmula para redondear las cifras al nuevo peso y una disposición para que el Banco de la República y la Superintendencia de Industria Comercio (SIC) pudieran sancionar a aquellos que abusaran en el proceso de fijar precios con la nueva denominación. En las ponencias a favor del proyecto surgió la incorporación de una estimación de los costos asociados a este (Vargas, 2002); no obstante, no se presentó un documento oficial que calculara los costos de esta medida. Este fue el primer proyecto de ley que incluyó de manera explícita una estimación de los costos asociados a la realización de este cambio.

Estos costos serían en principio la campaña educativa y la emisión de nuevos billetes y monedas. El Banco de la República aclaró que los costos de emisión de nuevos billetes y monedas no eran altos, pues estos sufren un proceso continuo de reemplazo; sin embargo, nunca presentó una 
cuantificación de los costos (beneficios) que él o la sociedad recibirían con la medida.

Un mes después que se hundiera en plenaria el anterior proyecto de ley, el 22 de julio de 2002 se presenta el segundo intento en este siglo para eliminar tres ceros del peso colombiano. El representante Luis Enrique Salas (Partido de la U) radicó el Proyecto de Ley 07 de 2002, "por medio de la cual se modifica la denominación de la moneda legal colombiana", muy similar en su motivación y contenido al anterior, aunque la forma cambió un poco (en vez de contar con doce artículos, tenía cinco). En términos de contenido, la mayor diferencia es que este implicaba la creación del nuevo peso de manera permanente y establecía un periodo de un año para la coexistencia del peso y el nuevo peso.

El primer artículo del proyecto creaba el nuevo peso con una equivalencia de un nuevo peso por 1000 pesos. El segundo artículo otorgaba poder liberatorio ilimitado al nuevo peso colombiano mientras coexistiera con el peso. El mismo artículo establecía que el periodo de coexistencia de las dos monedas sería de un año. El artículo tercero le otorgaba la función a la Junta del Banco de la República de realizar todas las tareas necesarias para garantizar que la conversión de pesos a nuevos pesos no implicara la pérdida del poder adquisitivo del dinero y, en especial, de los ingresos laborales. El cuarto artículo estaba encaminado a garantizar que la conversión de moneda extranjera al nuevo peso no se viera afectada ni distorsionada por el cambio de denominación de la moneda colombiana. El último artículo se refería a la fecha en que entraría en vigor la ley.

En la exposición de motivos no se presentó una argumentación nueva; es más, en el Proyecto de Ley 07 de 2002 se afirmó: "Hoy presentamos nuevamente esta iniciativa, seguros de que el nuevo Congreso la respaldará y dotará a la Junta Directiva del Banco de la República de una herramienta eficaz para el manejo del circulante y de la inflación". El proyecto fue aprobado en primer debate en la Comisión Tercera de la Cámara el 21 de mayo de 2003, pero fue archivado el 20 de junio de 2004 por Tránsito de Legislatura; es decir, no se presentó el interés de mantener la discusión de este proyecto.
Dos meses después del hundimiento del último proyecto, el 18 de agosto de 2004 se radica el Proyecto de Ley 121 con el mismo título del proyecto de 2002. En este caso, los autores del proyecto fueron los representantes Luis Enrique Salas (Partido de la U), Óscar Darío Pérez (Centro Democrático) y Ómar Armando Baquero (Partido Conservador Colombiano). Este proyecto tenía seis artículos, cinco de ellos idénticos al proyecto del 2002 y uno nuevo (el artículo 5), que incluyó unas disposiciones que permitían a la SIC proteger a los ciudadanos de especulaciones o alzas de precios que se dieran por el cambio de la denominación. Este artículo era algo similar al que se incluyó en el proyecto de 2000 durante el trámite en Cámara y Senado; asimismo, incluía de manera explícita cómo se deberían redondear los precios.

Por otro lado, en la exposición de motivos se elaboraron mucho mejor los beneficios y costos del proyecto cuando se comparan con los motivos de los proyectos anteriores. En esta ocasión, el Proyecto de Ley 121 de 2004 aclaró que "la principal razón por la que la normalización de la moneda puede ser benéfica tiene que ver con la disminución en los costos de transacción". El análisis de los costos y beneficios presentados en el proyecto son, según refiere Vargas (2002), los que ya se habían recogido en la propuesta de 2000 durante su curso por el Congreso, el 20 de junio de 2005. El proyecto fue archivado por Tránsito de Legislatura sin mediar debate.

Cuatro meses después, el 5 de octubre de 2005, se presenta el Proyecto de Ley 156 de 2005. Los representantes que radican el proyecto fueron los mismos que lo hicieron con el proyecto de 2004 , y el contenido es idéntico. La única diferencia se presentaba en una ampliación de los argumentos empleados en la motivación. El 3 de mayo de 2006, la Comisión Tercera aprobó el proyecto en primer debate. Posteriormente, el 22 de agosto de 2006 fue archivado tras su segundo debate.

El siguiente intento se dio el 27 de julio de 2010, cuando el senador Antonio del Cristo Guerra de La Espriella (Cambio Radical) presentó el Proyecto de Ley 34 de 2010. Por primera vez en el siglo se pasó un periodo legislativo completo sin 
un proyecto de ley que pretendiera la supresión de los tres ceros. Este nuevo proyecto era similar al presentado en el Proyecto de Ley 74 de 2000, pero contaba con quince artículos. El proyecto se distanciaba, en forma, de los proyectos presentados en 2004 y 2005 y retomaba la idea de que la circulación del nuevo peso fuese temporal.

Con respecto al Proyecto de Ley 74 del 2000, este implicó cuatro nuevos artículos y la eliminación de uno. Se incluye el artículo 11, que le da facultades a la SIC para promover la fijación de listas de precios en lugares visibles que mostraran los precios tanto en pesos como en nuevos pesos (este artículo es similar a uno que se introdujo en la discusión del proyecto de ley de 2000). También se incluye el artículo 12, donde se facultaba al Banco de la República a apropiar los recursos necesarios para realizar la difusión y pedagogía que requiriera la ley. El artículo 13 establecía un plazo de un año para que el Banco empezara a poner en circulación los billetes y las monedas de la nueva denominación. Finalmente, se incluyó el artículo 14, que le permitiría al Ministerio de Hacienda y Crédito Público establecer medidas de control durante el proceso de conversión de los pesos a nuevos pesos, para detectar dineros que provinieran de actividades dentro o fuera del país. El proyecto eliminó el último artículo que estaba presente en el radicado en el 2000 y que implicaba derogar las normas que estuviesen en contra de esta nueva.

En cuanto a la motivación, similar a los proyectos de 2004 y 2005, se emplean los cálculos de Vargas (2002) para justificar el proyecto, y en la exposición de motivos se aclaró que la finalidad de esta medida era "la simplificación en el manejo de las cantidades de la moneda nacional". El 19 de octubre de 2010, en primer debate en la Comisión Tercera del Senado, el proyecto fue aprobado. El 6 de diciembre de 2010, por primera vez, el Ministerio de Hacienda y Crédito Público expresó en una carta de tres líneas que no tenía observaciones de inconstitucionalidad o inconveniencia frente al proyecto de ley. Finalmente, tras una votación de 41 votos en contra y quince positivos (43 abstenciones), el proyecto es archivado el 4 de octubre de 2011, en segundo debate.
Casi cuatro años y medio después, el 31 de marzo de 2016 se radica el sexto proyecto de este siglo para redenominar la moneda nacional: Proyecto de Ley 156 de 2016, Cámara. Los autores de esta propuesta fueron los senadores Roy Barreras (Partido de la U), Mauricio Lizcano (Partido de la $U$ ), Armando Benedetti (Partido de la $U$ ), Sandra Villadiego (Partido de la U), Ángel Custodio Cabrera (Partido de la U), José David Name (Partido de la U), Andrés García Zuccardi (Partido de la U) y Miguel Amín (Partido de la U).

Este nuevo proyecto es similar a los presentados en 2002, 2004 y 2005; en otras palabras, frente al proyecto de 2010 se retoma la idea de que el cambio de la denominación de la nueva moneda sea permanente y no transitoria, como igualmente lo proponían el Proyecto de Ley 07 de 2002 y el Proyecto de Ley 34 de 2010. La principal diferencia entre el Proyecto de Ley 121 de 2004 (Cámara) y el 165 de 2005 es que establece un periodo de dos años, y no de uno, para que coexistan el peso y el nuevo peso. En la exposición de motivos se emplea un argumento poco ortodoxo e inédito en las discusiones anteriores: se adjunta una tabla en la que se presenta la cantidad de pesos colombianos necesarios para comprar una unidad monetaria de Argentina, Brasil, Chile, Estados Unidos, México, Haití, Perú, República Dominicana, Uruguay y Venezuela. El Proyecto de Ley 156 de 2016 argumenta:

En la muestra de once países, se verifica que el peso colombiano no supera en valor a ninguna de las monedas comparadas; esto, aunado a criterios como el de la globalización, que implica mayores inversiones en dólares, demuestra la necesidad de darle mayor valor al peso colombiano a través de la eliminación de tres dígitos; adicional a esto, en criterios prácticos, Presupuesto General de la Nación, así como los informes y estados financieros, pasarán a tener menos dígitos en cada una de sus cifras, lo que permitirá una mayor compresión de estas. 
Este argumento no tiene sustento técnico, pues el valor nominal de la tasa de cambio bilateral no representa el "valor de una moneda" (Banco de la República, s. f.). Ese fue el único argumento empleado para justificar el proyecto. Al igual que todas las iniciativas de este siglo, el proyecto de ley no tuvo éxito. El 20 de junio de 2016, sin mediar debate, fue archivado por Tránsito de Legislatura, tal como ocurrió con el proyecto radicado en 2004.

El séptimo intento de redenominación del peso durante este siglo se presenta un año y diez meses después: el 1 de abril de 2018, Mauricio Cárdenas Santamaría, ministro de Hacienda y Crédito Público, y los representantes Antonio del Cristo Guerra de La Espriella (Cambio Radical) y Jack Housni Jaller (Partido Liberal Colombiano) radicaron el Proyecto de Ley 231 de 2018, el primer proyecto del siglo presentado por un ministro de Hacienda.

Este proyecto tiene dieciséis artículos (el más largo hasta el momento) y su filosofía se asemeja un poco más a la de los proyectos presentados en 2000 y 2010. La estructura es algo diferente y por su extensión se organiza en títulos. El primero de ellos se concentra en la creación del nuevo peso; el segundo título, en el periodo de transición; el tercero, en el periodo de regreso del nuevo peso al peso, y en el último se ofrecen unas disposiciones generales. Dejando de lado la forma, el nuevo proyecto se parece a los presentados en 2000 y 2010 , donde se propone crear transitoriamente el nuevo peso, pero en este caso se establece un plazo de tres años para el periodo de transición y otros tres años más para regresar al peso como denominación legal (artículo 12).

En general, el proyecto presenta más detalle e incluye por primera vez, de manera explícita, la pérdida de poder liberatorio ilimitado del actual peso después del periodo de transición (artículo 9). Otra novedad es la de otorgar la facultad a la Junta del Banco de la República para definir un día a partir del cual los billetes y las monedas actuales podrán ser recibidos y cambiados por nuevos pesos en los establecimientos de crédito y en el Banco de la República; es más, el proyecto da la posibilidad de que estas fechas se fijen de manera diferencial de acuerdo con el valor del importe de cada billete o moneda.
Otra novedad por resaltar es que por primera vez se establece de manera explícita que la conversión de pesos a nuevos pesos y el regreso a pesos no puede tener costo para el consumidor o usuario.

Entrando en detalle frente al Proyecto de Ley de 2018, el primer título va del artículo 1 al 5 . Los primeros tres artículos corresponden al mismo contenido del artículo primero de los proyectos de ley de 2000 y 2010 . El cuarto artículo aclara cómo se realizará el redondeo a la segunda cifra decimal cuando se traduzcan los valores de pesos a nuevos pesos. Este artículo es similar al sexto artículo de los proyectos radicados en 2000 y 2010 , pero la diferencia es que en esta ocasión se presenta de manera más clara la forma en que se realizará el redondeo. En este último sentido, la redacción es más parecida a la de los proyectos de ley de 2004, 2005 y 2016. El artículo quinto, similar al artículo 11 del proyecto de 2010, les asigna a la SIC y a la Superintendencia Financiera la tarea de crear un régimen para proteger los derechos de los consumidores y usuarios.

El segundo título desarrolla las normas para el periodo de transición por medio de tres artículos. El artículo 6 establece un plazo de tres años para el periodo de transición de los billetes en pesos a los denominados en nuevos pesos, y de cuatro años para las monedas. Este artículo no es similar a alguno de los artículos de los proyectos anteriores. El artículo 7 es en parte similar al artículo 1 de los proyectos de 2000 y 2010 , al establecer el poder liberatorio ilimitado del nuevo peso. También es similar al artículo cuarto de los proyectos de 2000 y 2010, al estipular que las obligaciones contraídas en pesos se podrán pagar con nuevos pesos, y viceversa, durante el periodo de transición. El parágrafo de este artículo (similar al artículo 14 del proyecto de 2010) establece la necesidad de hacer controles al lavado de activos cuando se cambien los billetes expresados en pesos por aquellos en nuevos pesos. Por su parte, el artículo 8 establece que una vez inicie el periodo de transición, todos los precios y las obligaciones deberán expresarse en términos del nuevo peso, y así se recoge lo propuesto en al artículo 3 de las propuestas de 2000 y 2010. 
El tercer título reglamenta el fin del periodo de transición por medio de dos artículos. El artículo 9 es novedoso al establecer de manera explícita la pérdida de poder liberatorio ilimitado del peso una vez termine el periodo de transición. También incluye un parágrafo que implicaría que los billetes y las monedas expresadas en poder del público después del periodo de transición podrían ser cambiados a nuevos pesos en el Banco de la República y las instituciones de crédito. Estas transacciones también estarían sujetas a vigilancia para evitar el lavado de activos. El artículo 10 es similar a los artículos 8 y 9 de las propuestas de 2000 y 2010 , al proponer que todas las cifras que estén en pesos en leyes, decretos reglamentos, circulares, actos administrativos, etc., deberán ser convertidas a nuevos pesos.

El cuarto título, con seis artículos, discute disposiciones generales. El artículo 11 introduce una disposición inédita: se le otorga la facultad a la Junta del Banco de la República de fijar una fecha hasta la cual los billetes y monedas denominados en los actuales pesos solo podrán ser recibidos y cambiados por nuevos pesos en los establecimientos de crédito y en el banco central, aun durante el periodo de transición; es más, el proyecto da la posibilidad de que estas fechas se fijen de manera diferencial de acuerdo con el valor del importe de cada billete o moneda. El artículo 12 establece el regreso de la denominación de la moneda a peso nuevamente; en este sentido, lo expuesto se parece a lo propuesto en el séptimo artículo de los proyectos de 2000 y 2010 . No obstante, la diferencia en esta nueva propuesta es que establece que el regreso al peso se hará después de tres años de terminado el proceso de transición; es decir, tras seis años, el peso actual se volvería a llamar peso, pero tendría tres ceros menos.

El artículo 13 aclara que esta ley no afecta ni exime del pago de obligaciones previamente contraídas, similar a lo establecido en el artículo 4 de las propuestas de 2000 y 2010 . Los dos artículos siguientes hacen explícito que el cambio de unidades monetarias no puede generar costos para los consumidores o usuarios (artículo 14) y que las autoridades deben coordinarse para asegurar la adecuada aplicación de esta ley (artículo 15), lo cual no se había contemplado en las propuestas anteriores. El último artículo versa sobre la vigencia de la ley.

La exposición de motivos de este proyecto también presenta algunas innovaciones frente a los proyectos anteriores. Así, por ejemplo, se citan diversos estudios para sustentar los argumentos y se hace una revisión de los casos internacionales donde se ha presentado este tipo de cambio. Inicialmente, los autores analizan si el peso colombiano cumple cada una de las funciones de una moneda (depósito de valor, unidad de cuenta y medio de pago), pues de no hacerlo debería darse la redenominación. Argumentan que, dados los bajos y controlados niveles de inflación de los últimos dieciocho años, se puede concluir que la moneda colombiana preserva su papel de ser depósito de valor.

De igual forma, el proyecto argumenta que el peso colombiano cumple su papel como medio de pago, puesto que allí se encuentra la mayor parte de los ingresos, los ahorros y las deudas de los colombianos. Sin embargo, de acuerdo con los autores, el peso no cumple su papel de unidad de cuenta debido a que presenta algunas desventajas respecto a otras monedas, al tener un exceso de ceros. Aunque este motivo parece similar al presentado en el Proyecto de Ley 156 de 2016, se distingue al establecer que la importancia de esa diferencia en el número de dígitos radica en el efecto psicológico que puede tener en los agentes económicos, y no en el valor del peso colombiano respecto a otras monedas.

Posteriormente, los autores describen los beneficios y costos de la conversión monetaria. Similar a los proyectos anteriores, se enuncian los siguientes beneficios: la reducción de los costos de transacción, costos de impresión, costos de publicación y los requerimientos de almacenamiento computacional, así como la simplificación de la contabilidad y el manejo de cifras. Por un lado, se diferencia de los proyectos anteriores al incluir el efecto que tendría sobre la ilusión monetaria, la cual disminuiría, y con ello, las presiones inflacionarias; por otro lado, no incluye algunos beneficios nombrados anteriormente, como la interpretación de este cambio en cuanto un compromiso para mantener la 
estabilidad de los precios o la posibilidad de identificar recursos derivados de actividades ilegales.

Para el caso de los costos, en este proyecto se presenta una actualización del valor estimado que tendría que asumir el país por el cambio de las monedas y los billetes. El último cálculo se presentó en el Proyecto de Ley 121 de 2004 y el Proyecto de Ley 165 de 2005, y se basaba en la estimación que realizó el Banco de la República en 2001. De igual forma, allí se reconoce que habría que presupuestar recursos para una campaña de divulgación, aunque no se incluye un valor aproximado de esta, como sí lo hace el Proyecto de Ley 121 de 2004. Tampoco se tiene en cuenta el costo que asumirán los agentes económicos para actualizar los softwares, la papelería y los "costos de menú" a los nuevos pesos, como sí lo incluye el Proyecto de Ley 121 de 2004. Por último, se clasifican los motivos de otros países que han experimentado la redenominación en dos grupos: como medida de ajuste macroeconómico o como medida para alcanzar el balance entre la moneda local y las monedas de la región, simplificando el manejo de las cantidades. Según todo lo mencionado, Colombia formaría parte de este segundo grupo de países si el proyecto es aprobado.

Los autores del proyecto agregan que Colombia debería eliminar los tres ceros porque su alta denominación es producto de procesos inflacionarios pasados, y la alta denominación actual entorpece temas operativos y de eficiencia.
Sustentan parte de lo anterior al referir que el país se encuentra en los primeros puestos del ranking de países con billetes de más alta denominación, junto a países que no comparten las mismas condiciones económicas; afirman que "los niveles de inflación de nuestro país son inferiores a los de la mayoría de países del cuadro". No obstante, de los dieciocho países que componen el ranking (incluido Colombia), la inflación de 2017 de Colombia solo estuvo por debajo de seis países $(35,3 \%)$ y la inflación de 2018 estimada por el Fondo Monetario Internacional para el país está por debajo de diez países $(58,8 \%)$.

\section{CONCLUSIONES}

En la sección anterior se discutieron en detalle los sietes proyectos de redenominación que se han radicado en el Congreso durante el siglo XXI (tabla 1). De la discusión se puede evidenciar que, si bien existen muchas similitudes entre todos los proyectos, estos se pueden clasificar en dos grupos: los proyectos de redenominación permanente al nuevo peso y los que no. Los primeros se caracterizan por ser cortos e implicar un paso del peso al nuevo peso de manera permanente; estos proyectos comprenden los radicados en 2002, 2004, 2005 y 2016. En la figura 1 se presenta de manera esquemática un resumen del análisis realizado. Es claro que no

Tabla 1.

Resumen de proyectos de ley para eliminar ceros durante el siglo XXI

\begin{tabular}{|c|c|c|c|c|c|c|c|c|}
\hline Año & Proyecto de Ley & $\begin{array}{c}\text { Número } \\
\text { de } \\
\text { debates } \\
\text { en } \\
\text { Senado }\end{array}$ & $\begin{array}{c}\text { Número } \\
\text { de } \\
\text { debates } \\
\text { en } \\
\text { Cámara }\end{array}$ & Razón de archivo & $\begin{array}{c}\text { Fecha de } \\
\text { archivo }\end{array}$ & $\begin{array}{c}\text { Número } \\
\text { de } \\
\text { artículos }\end{array}$ & $\begin{array}{c}\text { Nueva } \\
\text { denominación }\end{array}$ & $\begin{array}{c}\text { Años de } \\
\text { ajuste }\end{array}$ \\
\hline 2000 & 74 de 2000 (Senado) & 2 & 2 & Archivado en debate & Jun.-18-2002 & 12 & Transitoria & Indefinidos \\
\hline 2002 & 07 de 2002 (Cámara) & 0 & 1 & Tránsito de legislatura & Jun.-20-2004 & 5 & Indefinida & 1 \\
\hline 2004 & 121 de 2004 (Cámara) & 0 & 0 & Tránsito de legislatura & Jun.-20-2005 & 6 & Indefinida & 1 \\
\hline 2005 & 165 de 2005 (Cámara) & 0 & 1 & Archivado en debate & $\begin{array}{c}\text { Ago.-22- } \\
2006\end{array}$ & 6 & Indefinida & 1 \\
\hline 2010 & 34 de 2010 (Senado) & 2 & 0 & Archivado en debate & Oct.-4-2011 & 15 & Transitoria & Indefinidos \\
\hline 2016 & 156 de 2016 (Cámara) & 0 & 0 & Tránsito de legislatura & Jun.-20-2016 & 6 & Indefinida & 2 \\
\hline 2018 & 231 de 2018 (Cámara) & N. A. & N. A. & N. A. & N. A. & 16 & Transitoria & 3 \\
\hline
\end{tabular}

Fuente: elaboración de los autores. 
ha existido mucha innovación en los fallidos proyectos de ley de este grupo.

El segundo conjunto corresponde a los proyectos de redenominación transitoria al nuevo peso; se caracterizan por tener muchos más artículos e implicar dos periodos: uno de transición del peso actual al nuevo peso y otro del nuevo peso de regreso a la denominación de peso. En la figura 2 se presenta de manera esquemática un resumen del análisis realizado para este grupo, en el cual ha existido un poco más de innovación que en el otro conjunto. Claramente, el proyecto de ley de 2018 presenta la mayor cantidad de nuevas disposiciones frente a los proyectos anteriores; no obstante los nuevos detalles de este proyecto, donde parece existir la necesidad de aclarar más detalles que en los otros proyectos no se encontraban, no parece existir mucho espacio para innovar frente a los proyectos anteriores.

Por el lado de la exposición de motivos sí parece haber posibilidades para trabajar. Los documentos de Ministerio de Hacienda y Crédito Público (2001) y Vargas (2002) han sido los únicos intentos para cuantificar de manera integral el impacto de esta medida. Si bien el séptimo y último proyectos de ley presentan los costos de cambiar las monedas y los billetes, esta cuantificación es insuficiente para sopesar los beneficios y costos de la transacción propuesta.

El último proyecto de ley debería incluir una cuantificación actualizada de los recursos que se necesitarían para realizar la campaña educativa; de igual forma, debería estimar el costo que tendría para las empresas el cambio en el sistema contable, la actualización de precios, la impresión de nueva papelería, etc. Además, si bien se menciona el potencial impacto psicológico que tendrá el cambio en el comportamiento microeconómico de los agentes, ciertamente esto no es medido. Ejercicios como el propuesto por Dkazote et al. (2010), Tyska y Przybyszewski (2006) o Amado et al. (2007) intentan llenar ese vacío. Las primeras dos fuentes evalúan cómo los mecanismos psicológicos modifican la fuerza del efecto de la ilusión monetaria, en tanto la tercera estudia los procesos cognitivos que se dan durante la transacción de la denominación de la moneda. Por último, deberían actualizarse los beneficios que había cuantificado el Ministerio de Hacienda y Crédito Público (2001) en términos de reducción de costos transaccionales.

Figura 1

Estructura y relación de los proyectos de redenominación permanente al nuevo peso durante el siglo XXI

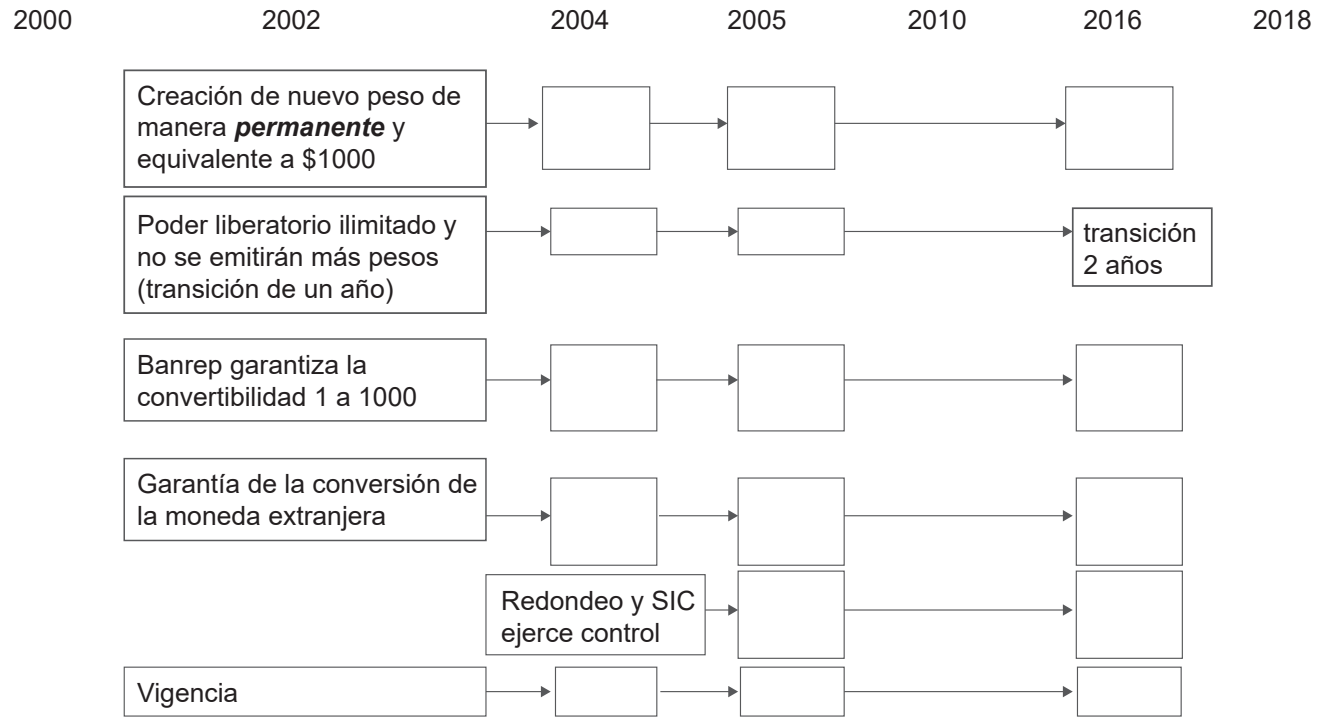

Fuente: elaboración de los autores. 
Figura 2.

Estructura y relación de los proyectos de redenominación transitoria al nuevo peso durante el siglo XXI

\begin{tabular}{|l|l|}
\hline $\begin{array}{l}\text { Creación de nuevo peso(N\$) de manera } \\
\text { transitoria y equivalente a \$1000 y con } \\
\text { poder liberatorio ilimitado }\end{array}$ \\
\hline $\begin{array}{l}\text { Seguir emitiendo pesos durante la } \\
\text { transición }\end{array}$ \\
\hline Todos los precios se expresán en $\mathrm{N} \$$ \\
\hline expresarán en $\mathrm{N} \$$ \\
moneda garantiza la conversión de la \\
\hline $\begin{array}{l}\text { Regreso al peso cuando se crea } \\
\text { conveniente }\end{array}$ \\
\hline Reglas para regresar al \$ son las mismas
\end{tabular}

Fuente: elaboración de los autores.

\section{REFERENCIAS}

1. Alonso, J., Estrada, D. y Gonzales, A. (2016). Elementos para la discusión de la eliminación de tres ceros en el peso colombiano. En J. Escobar (Ed.), Análisis socioeconómicos: un aporte desde la academia a la actividad legislativa (pp. 29-55). Bogotá: Senado de la República de Colombia.

2. Amado, S., Teközel, M., Topsever, Y., Ranyard, R., Del Missier, F. y Bonini, N. (2007). Does “000,000” matter? Psychological effects of Turkish monetary reform. Journal of Economic Psychology, 28(2), 154-169.

3. Avella, M. (1987). Pensamiento y política monetaria en Colombia (1886-1945). Bogotá: Banco de la República.

4. Banco de la República de Colombia (2015). Informe de política monetaria. Recuperado de http://www. banrep.gov.co/economia/pli/dmjd_feb_2015.pdf

5. Banco de la República de Colombia (s. f.). ¿Qué es la tasa de cambio? Recuperado de http://www.banrep. gov.co/es/contenidos/page/qu-tasa-cambio

6. Cámara de Representantes de Colombia (2002). Proyecto de Ley 07 de 2002, por medio de la cual se modifica la denominación de la moneda legal colombiana. Recuperado de http://www.imprenta.gov.co/ gacetap/gaceta.mostrar_documento?p_tipo $=05 \&$ __numero $=07 \&$ p_consec $=5101$

7. Cámara de Representantes de Colombia (2004). Proyecto de Ley 121 de 2004, por medio de la cual se modifica la denominación de la moneda legal colombiana. Recuperado de http:/www.imprenta.gov.co/ gacetap/gaceta.mostrar_documento?p_tipo $=05 \&$ p_numero $=121 \&$ p_consec $=8875$

8. Cámara de Representantes de Colombia (2005). Proyecto de Ley 156 de 2005, por medio de la cual se modifica la denominación de la moneda legal colombiana. Recuperado de http://www.imprenta.gov.co/ gacetap/gaceta.mostrar_documento?p_tipo $=05 \&$ p_numero $=165 \&$ p_consec $=11748$ 
9. Congreso de la República de Colombia (1821). Constitución Política de la República de Colombia de 1821. Recuperado de http://biblio.juridicas.unam.mx/libros/5/2212/6.pdf

10. Dzokoto, V., Mensah, E., Twum-Asante, M. y Opare-Henaku, A. (2010). Deceiving our minds: A qualitative exploration of the money illusion in post-redenomination Ghana. Journal of Consumer Policy, 33(4), 339-353.

11. El País (2018, 28 de febrero). Los pros y contras del proyecto que busca quitarle ceros al peso. Recuperado de http://www.elpais.com.co/economia/los-pros-y-contras-del-proyecto-que-busca-quitarle-ceros-al-peso. html

12. Ibáñez, J. (1990) El billete del Banco de la República como moneda legal colombiana. En Banco de la República (Ed.), El Banco de la República: antecedentes, evolución y estructura (pp. 33-65). Bogotá: Banco de la República.

13. Ministerio de Hacienda de Colombia (2001). Estimación de la reducción en los costos de transacción por la sustitución monetaria. Bogotá: autor.

14. Mosley, L. (2005). Dropping zeros, gaining credibility? Currency redenomination in developing nations. Recuperado de https://www.unc.edu/ lmosley/APSA\%202005.pdf

15. Odior, E. y Shodeinde, A. (2013). Currency restructuring exercise in Nigeria: Overview and potential implications. International Journal of Business and Social Research, 3(6), 8-16.

16. Pérez, H. (2008). La reconversión del peso colombiano (tesis de posgrado). Bogotá: Universidad de La Sabana. Recuperado de http://intellectum.unisabana.edu.co/bitstream/handle/10818/5895/127805. pdf? sequence $=1$

17. Portafolio (2018, 24 de febrero). Gobierno volverá a intentar eliminar los tres ceros de los billetes colombianos. Recuperado de http:/www.portafolio.co/economia/gobierno/gobierno-apoya-eliminacion -de-los-tres-ceros-de-los-billetes-colombianos-514616

18. Revista Dinero (2018a, 23 de febrero). La Fiscalía se une a la petición del nuevo peso. Recuperado de http://www.dinero.com/pais/articulo/fiscalia-pide-eliminar-ceros-al-peso/255708

19. Revista Dinero (2018b, 25 de febrero). Minhacienda apoya propuesta de eliminar tres ceros del peso. Recuperado de http://www.dinero.com/economia/articulo/minhacienda-apoya-propuesta-deeliminar-tres-ceros-del-peso/255729

20. Senado de la República de Colombia (2000). Proyecto de Ley 74 de 2000, por medio de la cual se modifica la denominación de la moneda legal colombiana, en desarrollo del numeral 13 del artículo 150 de la Constitución Política. Recuperado de http://www.imprenta.gov.co/gacetap/gaceta. mostrar_texto?p_tipo $=05 \&$ p_numero $=74 \&$ __consec $=296$

21. Senado de la República de Colombia (2010). Proyecto de Ley 34 de 2010, por medio de la cual se modifica la denominación de la moneda legal colombiana, en desarrollo del numeral 13 artículo 150 de la Constitución Política. Recuperado de http://www.imprenta.gov.co/gacetap/gaceta. mostrar_documento?p_tipo $=05 \&$ p_numero $=34 \&$ __consec $=26217 \#$ ftn2

22. Senado de la República de Colombia (2016). Proyecto de Ley 156 de 2016, por medio de la cual se modifica la denominación de la moneda legal colombiana y se dictan otras disposiciones. Recuperado de http://www. imprenta.gov.co/gacetap/gaceta.mostrar_documento?p_tipo $=05 \&$ p_numero $=156 \&$ __consec $=44122$

23. Senado de la República de Colombia (2018). Proyecto de Ley 231 de 2018, por medio del cual se crea una nueva unidad monetaria y unidad de cuenta del país, en desarrollo del numeral 13 del artículo 150 de la Constitución Política. El Congreso de Colombia. Recuperado de https://vlex.com.co/vid/ proyecto-ley-231-2018-714874817 
24. Torres, G. (1980). Historia de la moneda en Colombia. Medellín: Fondo Rotatorio de Publicaciones.

25. Tyszka, T. y Przybyszewski, K. (2006). Cognitive and emotional factors affecting currency perception. Journal of Economic Psychology, 27(4), 518-530.

26. Urrutia, M. (2001). Resultados recientes del debate sobre regímenes cambiarios. Reportes del Emisor, 25. Recuperado de http://www.banrep.gov.co/sites/default/files/publicaciones/archivos/25_0.pdf

27. Vargas, J. (2002). Sustitución monetaria en Colombia: costos y beneficios [Borradores de Economía 214]. Bogotá: Banco de la República. 